\title{
Light Extraction Efficiency Enhancement of Colloidal Quantum Dot Light-Emitting Diodes Using Large-Scale Nanopillar Arrays
}

\author{
Xuyong Yang, Kapil Dev, Jianxiong Wang, Evren Mutlugun, Cuong Dang, Yongbiao Zhao, \\ Shuwei Liu, Yuxin Tang, Swee Tiam Tan, Xiao Wei Sun,* and Hilmi Volkan Demir*
}

A colloidal quantum dot light-emitting diode (QLED) is reported with substantially enhanced light extraction efficiency by applying a layer of largescale, low-cost, periodic nanopillar arrays. Zinc oxide nanopillars are grown on the glass surface of the substrate using a simple, efficient method of non-wetting templates. With the layer of $\mathrm{ZnO}$ nanopillar array as an optical outcoupling medium, a record high current efficiency (CE) of $26.6 \mathrm{~cd} / \mathrm{A}$ is achieved for QLEDs. Consequently, the corresponding external quantum efficiency (EQE) of 9.34\% reaches the highest EQE value for green-emitting QLEDs. Also, the underlying physical mechanisms enabling the enhanced light-extraction are investigated, which leads to an excellent agreement of the numerical results based on the mode theory with the experimental measurements. This study is the first account for QLEDs offering detailed insight into the light extraction efficiency enhancement of QLED devices. The method demonstrated here is intended to be useful not only for opening up a ubiquitous strategy for designing high-performance QLEDs but also with respect to fundamental research on the light extraction in QLEDs.
X. Yang, Dr. K. Dev, Dr. J. Wang, Dr. E. Mutlugun,

Dr. C. Dang, Dr. Y. Zhao, S. Liu, Dr. S. T. Tan,

Prof. X. W. Sun, Prof. H. V. Demir

Luminous! Center of Excellence for

Semiconductor Lighting and Displays

School of Electrical and Electronic Engineering

School of Physical and Mathematical Sciences

Nanyang Technological University

Nanyang Avenue

Singapore 639798, Singapore

E-mail: EXWSun@ntu.edu.sg; VOLKAN@stanfordalumni.org

Dr. E. Mutlugun, Prof. H. V. Demir

School of Physical and Mathematical Sciences

Nanyang Technological University

Nanyang Avenue

Singapore 639798, Singapore

Dr. E. Mutlugun, Prof. H. V. Demir

Department of Electrical and Electronics Engineering

Department of Physics

UNAM - Institute of Materials Science and Nanotechnology

Bilkent University

Bilkent, Ankara 06800, Turkey

Dr. Y. Tang

School of Materials Science and Engineering

Nanyang Technological University

Nanyang Avenue

Singapore 639798, Singapore

DOI: 10.1002/adfm.201400190

\section{Introduction}

Colloidal quantum dots (QDs) offer greatly advantageous features including high emission efficiency, ${ }^{[1,2]}$ narrow emission linewidth, ${ }^{[3,4]}$ tunable emission wavelength, ${ }^{[5,6]}$ and low-cost solution-processing, ${ }^{[7]}$ making them one of the most promising materials for lighting-emitting diodes (LEDs). Recently, current-driven quantum dot lighting-emitting diodes (QLEDs) have been intensively investigated as potential candidates for next-generation lighting and displays, and QLEDs are now competing with state-of-the-art organic LEDs (OLEDs) as well as other emerging solid-state lighting and display technologies. ${ }^{[8-16]}$ However, even though a $90 \%$ internal quantum efficiency (IQE) has been realized by using highly efficient QD emitters, ${ }^{[8]}$ the external quantum efficiency (EQE) of QLEDs has been severely

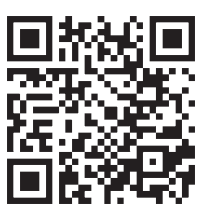
limited and is encountering a fundamental bottleneck due to the low light extraction efficiency (LEE). ${ }^{[9]}$

The EQE of QLEDs depends not only on IQE but also on the external outcoupling efficiency $\eta_{\text {oc }}$ (which is defined as the ratio of the total number of photons coupled out in the forward direction to the total number of photons generated within the QD emissive layer). For a conventional OLED in a planar waveguidelike structure, most of the generated light $(\sim 80 \%)$ is trapped in substrate modes and waveguided modes, or is coupled to surface plasmon modes associated with metallic electrodes, and finally only around $20 \%$ of internally generated photons can be extracted into air. ${ }^{[17,18]}$ Given the structural similarity between QLEDs and OLEDs, it can be expected that the $\eta_{\text {oc }}$ of QLEDs is quite low as well. The low $\eta_{\mathrm{oc}}$ in QLEDs leaves much room for device performance improvement. Furthermore, the QLEDs also possess its own speciality in device architecture. Currently, the QLED design using an inverted structure, comprising a few monolayers of QDs sandwiched between an inorganic metal oxide electron transport layer (ETL) and an organic hole transport layer (HTL), has risen to prominence as a result of its record efficiency ${ }^{[8]}$ and brightness. ${ }^{[19]}$ In such an inverted QLED architecture, the total internal reflection (TIR) would change to a great extent because of the large difference in refractive indices between organics $(\mathrm{n} \sim 1.75)^{[20]}$ and inorganic materials (QDs 
and inorganic charge transport materials). On the other hand, when placed in a resonant microcavity, the electroluminescence (EL) intensity of a QLED could undesirably enhanced at different levels depending on the cavity design since the emission linewidth of QDs is much narrower compared to many organic dye emitters. Therefore, in-depth studies on the light extraction from QLEDs are essential to improve their device performance and understand the critical conditions.

Here, we report a quantum dot light-emitting diode with substantially enhanced light outcoupling efficiency through applying a layer of large-scale, periodic zinc oxide nanopillar arrays. Here the uniform nanopillar arrays are obtained by a simple and efficient Pattern Replication In Non-wetting Templates (PRINT) technique. The resulting QLEDs with the ordered zinc oxide nanostructure serving as the light outcoupling medium exhibit superior performance compared to that of the reference QLEDs without light extraction features in terms of all important figure-of-merits including the maximum luminance, current efficiency (CE) and EQE values of $54200 \mathrm{~cd} / \mathrm{m}^{2}$, $26.6 \mathrm{~cd} \mathrm{~A}^{-1}$ and $9.34 \%$, respectively. To our knowledge, the CE of $26.6 \mathrm{~cd} \mathrm{~A}^{-1}$ is the record high current efficiency value for QLEDs and the EQE of $9.34 \%$ corresponds to the highest reported EQE value for green-emitting QLEDs. The relevant mechanisms enabling the enhanced light extraction efficiency are further discussed in details, which were found to lead to an excellent agreement between the simulation results and the experimental data, providing us with useful insight important for QLEDs.

\section{Results and Discussion}

The resulting QLED comprises a multilayer thin film architecture of ITO/ZnO NPs/QDs/4,4'-N,N'-dicarbazole-biphenyl
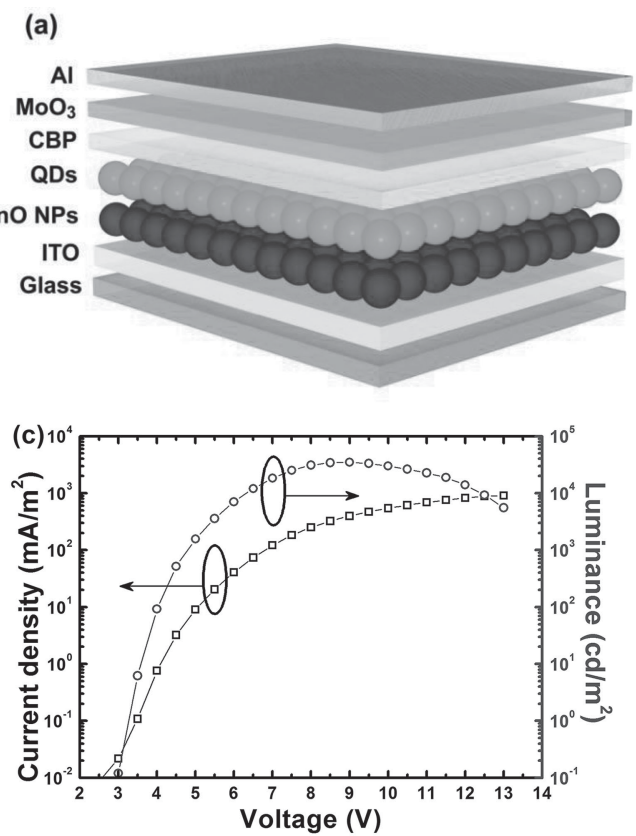

(CBP) $/ \mathrm{MoO}_{3} / \mathrm{Al}$, as shown in Figure $1 \mathrm{a}$, where $\mathrm{MoO}_{3}$ was used as the hole injection layer (HIL), CBP as the HTL, CdSe/ZnS core-shell structured QDs as the emissive layer (EML), ZnO NP film as the ETL spin-casted from ZnO NPs in ethanol solution, and $\mathrm{Al}$ as the anode. Figure $1 \mathrm{~b}$ presents the EL spectrum of our optimized QLED with a $20 \mathrm{~nm}$ thick QD layer and a $40 \mathrm{~nm}$ thick ZnO NP layer (the reference device). The pure green QD emission at a peak wavelength of $\sim 510 \mathrm{~nm}$ and with a full-width-at-half-maximum (FWHM) of $32 \mathrm{~nm}$ illustrates the highly efficient recombination of electrons and holes in the QD emissive layer. A corresponding image of the QLED output was recorded at the luminance of $250 \mathrm{~cd} / \mathrm{m}^{2}$, which displays uniform, bright and saturated green emission (inset of Figure $1 \mathrm{~b}$ ). The typical current density and luminance curves as a function of applied voltage for the device are depicted in Figure 1c. This shows that the maximum luminance reaches $34,900 \mathrm{~cd} / \mathrm{m}^{2}$ and the turn-on voltage $\left(\mathrm{V}_{\mathrm{T}}\right)$ of $3 \mathrm{~V}$ is relatively low, suggesting a small barrier height for the charge injection into the QDs. Here the $\mathrm{V}_{\mathrm{T}}$ is defined as the voltage at which the luminance is $1 \mathrm{~cd} \mathrm{~m}^{-2}$. The current efficiency-external quantum efficiencycurrent density (CE-EQE-J) characteristics of the QLED exhibit a high device efficiency (Figure 1d), with a maximum CE and EQE of $17.6 \mathrm{~cd} / \mathrm{A}$ and $6.17 \%$, respectively, which are the highest reported values for green QLEDs to date. ${ }^{[19]}$

In optimizing the device performance, it is found that, besides the important effects of QD and ZnO NP layer thickness, ${ }^{[8,21]}$ the performance of the resulting QLEDs is also strongly dependent on the annealing temperature of $\mathrm{ZnO} \mathrm{NP}$ film. As shown in Figure 2a, for the QLED with a $20 \mathrm{~nm}$ thick QD layer and a $40 \mathrm{~nm}$ thick $\mathrm{ZnO} \mathrm{NP}$ layer, increasing $\mathrm{ZnO}$ annealing temperature from 90 to $180{ }^{\circ} \mathrm{C}$, the $\mathrm{V}_{\mathrm{T}}$ of device shows a steady decrease from 4.5 to $3.0 \mathrm{~V}$ and the peak luminance of $34900 \mathrm{~cd} / \mathrm{m}^{2}$ is obtained at the annealing temperature
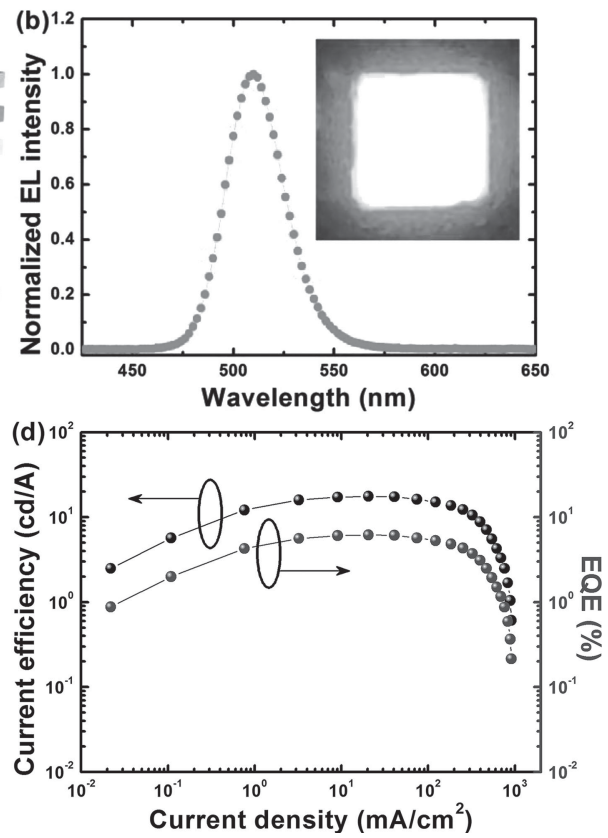

Figure 1. (a) Schematic of QLED configuration. (b) EL spectrum of the resulting QLED at an applied voltage of 6 V. Inset: the corresponding photograph of the QLED under operation with a pixel size of $3 \mathrm{~mm} \times 3 \mathrm{~mm}$. (c) Current density-voltage (J-V) and luminance-voltage (L-V) characteristics. (d) Current efficiency and external quantum efficiency versus current density (CE-EQE-J characteristics). 

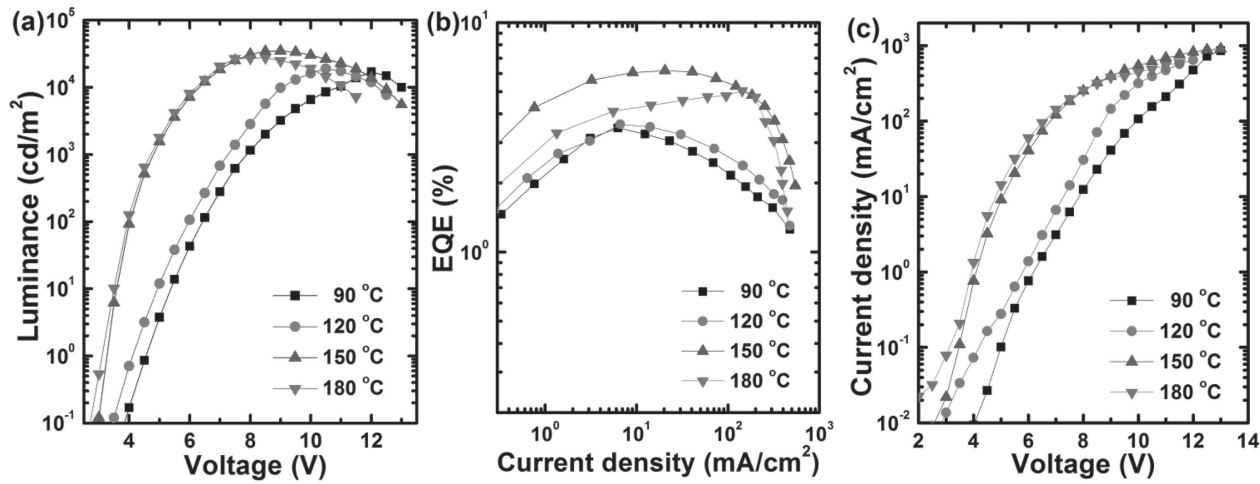

Figure 2. (a) Luminance-voltage (L-V), (b) external quantum efficiency-current density (EQE-J), and (c) current density-voltage (J-V) characteristics for QLEDs with $\mathrm{ZnO}$ nanoparticle films annealed at different temperatures from 90 to $180^{\circ} \mathrm{C}$.

of $150{ }^{\circ} \mathrm{C}$. In Figure $2 \mathrm{~b}$, it can be observed that the maximum EQE level of $6.17 \%$ is also obtained at $150{ }^{\circ} \mathrm{C}$. This is enabled by enhancing the electron transport in $\mathrm{ZnO}$ nanoparticle films, which is confirmed by the J-V curves of these devices in Figure 2c. Meanwhile, it can be also noted that there is no obvious change in the J-V curves between 150 and $180{ }^{\circ} \mathrm{C}$ annealing and for $180{ }^{\circ} \mathrm{C}$ annealing, however, the device EL performance becomes slightly worse, suggesting that $150{ }^{\circ} \mathrm{C}$ is the optimal annealing temperature in our study.

With a structure similar to OLEDs, the LEE of QLEDs is significantly limited due to reflection from the glass-air interface. It has been previously demonstrated that photonic crystal nanostructures can be used above the glass surface for enhancing the LEE of an OLED device where the trapped light within the QLED escapes into the air as a result of Bragg diffraction and scattering from the nanostructures on the glass surface. ${ }^{[22-24]}$ Among them, $\mathrm{ZnO}$ nanostructures are promising materials as light outcoupling medium because of their high index and excellent optical transparency as well as easy solution fabrication process. ${ }^{[25-27]}$ For our case, the light extraction enhancement in QLEDs is investigated by placing ordered $\mathrm{ZnO}$ nanopillar arrays onto the glass surface of ITO-glass substrates, as shown in Figure 3a. Our method is quite simple and efficient, which allows for high-quality $\mathrm{ZnO}$ nanostructures to be directly imprinted with precursor solutions. Figure $3 \mathrm{~b}$ briefly illustrates the fabrication process for the $\mathrm{ZnO}$ nanopillar arrays used here. A polydimethylsiloxane (PDMS) mold was first prepared using the patterned silicon master substrate (a thin Ni layer of $20 \mathrm{~nm}$ was deposited on the PDMS mold as an anti-adhesion layer). Under an applied pressure of $\sim 3 \mathrm{kPa}$, the PDMS mold was pressed on the glass surface of patterned ITO substrate, which was pre-coated with $\mathrm{ZnO}$ precursor solution composed of $\mathrm{Zn}(\mathrm{Ac})_{2}$, DEA and water, allowing for the formation of the sol-gel ZnO nanoparticles. The ITO-glass substrate was then annealed at $80^{\circ} \mathrm{C}$ for removing solvent. Subsequently, the mold was peeled off, leaving behind a periodical nanopillar array. Finally, the as-prepared substrate was annealed at $180{ }^{\circ} \mathrm{C}$ for 15 min. The PRINT process was performed in ambient environment. The large-scale $\mathrm{ZnO}$ nanopillars were designed of equal diameter and height $(200 \mathrm{~nm})$, and the total thickness of the formed $\mathrm{ZnO}$ nanostructured film was $\sim 200 \mathrm{~nm}$. Note that prior to the fabrication of $\mathrm{ZnO}$ nanostructures, a $\mathrm{ZnO}$ thin film (a)

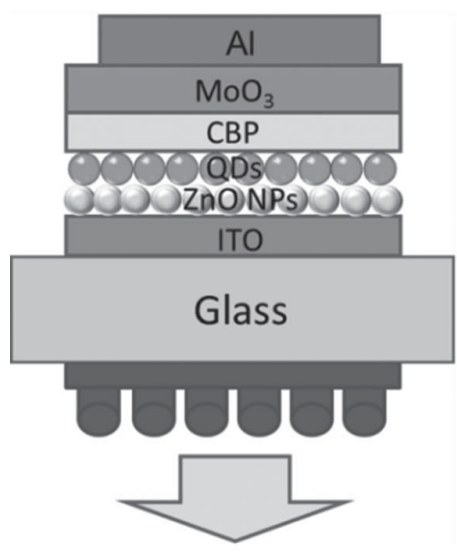

(b)

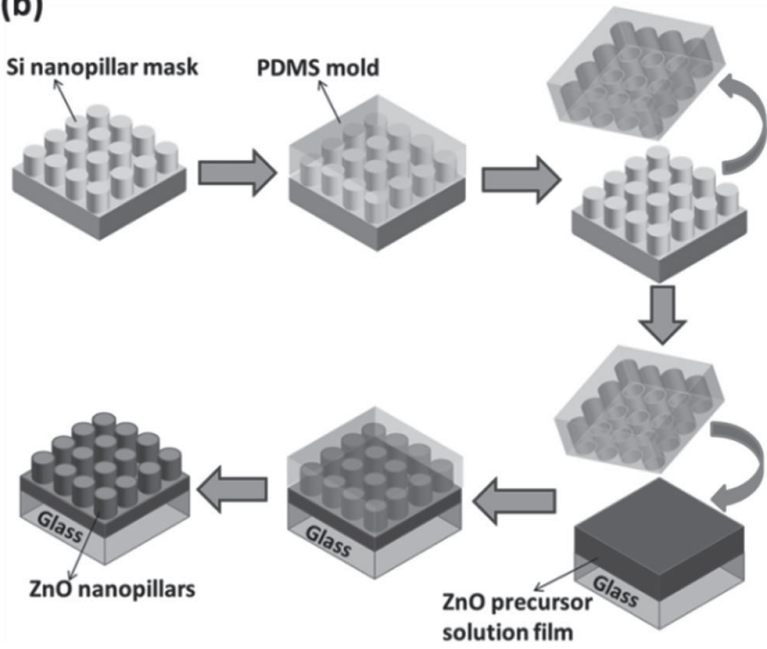

Figure 3. (a) Schematic illustration of the QLED with $\mathrm{ZnO}$ nanopillar arrays. (b) PRINT process used to fabricate the $\mathrm{ZnO}$ nanopillar arrays on the glass surface of ITO-glass substrate. 


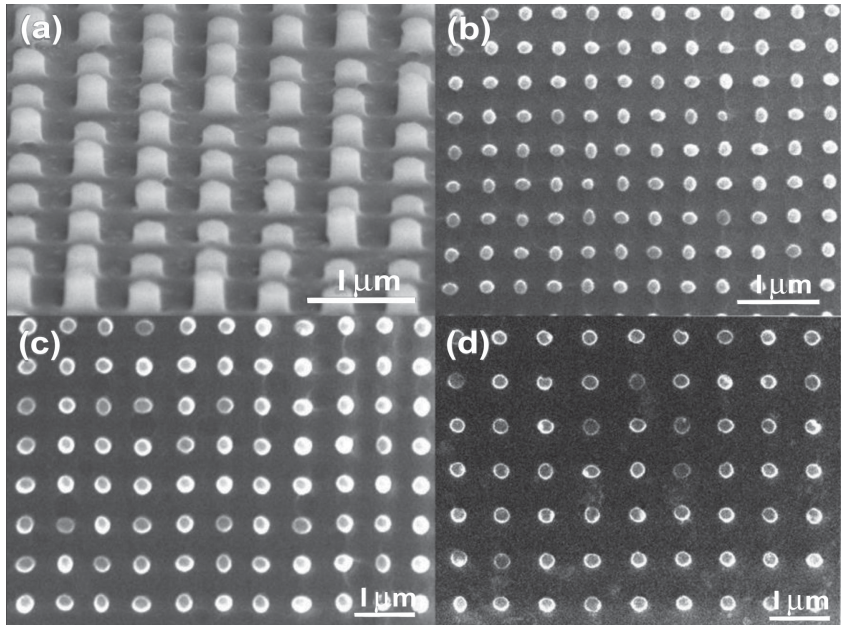

Figure 4. (a) $60^{\circ}$ side-view FESEM image of ordered $\mathrm{ZnO}$ nanopillar arrays with a pitch of $600 \mathrm{~nm}$. The top view SEM images of the $\mathrm{ZnO}$ nanopillar arrays with a pitch of (b) $400 \mathrm{~nm}$, (c) $600 \mathrm{~nm}$, and (d) $800 \mathrm{~nm}$.

of $20 \mathrm{~nm}$ was first deposited on the glass surface as a $\mathrm{ZnO}$ seed layer by RF magnetron sputtering for obtaining high-quality $\mathrm{ZnO}$ nanopillar arrays.

Figure 4 shows the enlarged tilt-view field-emission scanning electron microscopy (FE-SEM) and plane-view scanning electron microscopy (SEM) images for the as-prepared $\mathrm{ZnO}$ nanopillar arrays grown on the glass surface of the QLED substrate. In Figure 4a, it can be clearly observed that the imprinted $\mathrm{ZnO}$ nanopillar arrays exhibit uniform size distribution with a nanopillar array pitch of $600 \mathrm{~nm}$. The enhancement of light outcoupling by photonic crystals is based on Bragg diffraction and scattering. It is therefore desirable to choose an approximate nanopattern period that is comparable to the wavelength of the light for maximized light outcoupling. In our case, the pitches of $\mathrm{ZnO}$ nanopillar arrays can be conveniently and accurately controlled by adjusting the size of the patterned silicon master substrate. As shown in Figures $4 b-d$, such different pitches of the periodic $\mathrm{ZnO}$ nanopillar arrays were obtained and measured by the SEM image to be 400,600, and $800 \mathrm{~nm}$, respectively.

Figure 5a shows the EL spectra for the reference device without $\mathrm{ZnO}$ nanopillar arrays and the devices with $\mathrm{ZnO}$ nanopillar arrays measured using an integrating optical sphere at a current density of $100 \mathrm{~mA} / \mathrm{cm}^{2}$. Comparisons of these integral EL spectra exhibit no significant difference in the EL peak positions (at $\sim 510 \mathrm{~nm}$ ) for these devices. However, EL intensities obtained from the QLEDs with the ZnO nanopillars were obviously enhanced, especially for the device with the $\mathrm{ZnO}$ nanopillar array pitch of $600 \mathrm{~nm}$. Figure $5 \mathrm{~b}$ presents the normalized angular emission intensity for these devices. It can be seen that the reference device and the device with the $\mathrm{ZnO}$ pillar array pitch of $400 \mathrm{~nm}$ show similar angular distribution characteristics with an approaching Lambertian emission pattern, which implies that the diffraction effect for the device with $400 \mathrm{~nm}$ pitch ZnO pillars is not very significant. The QLEDs
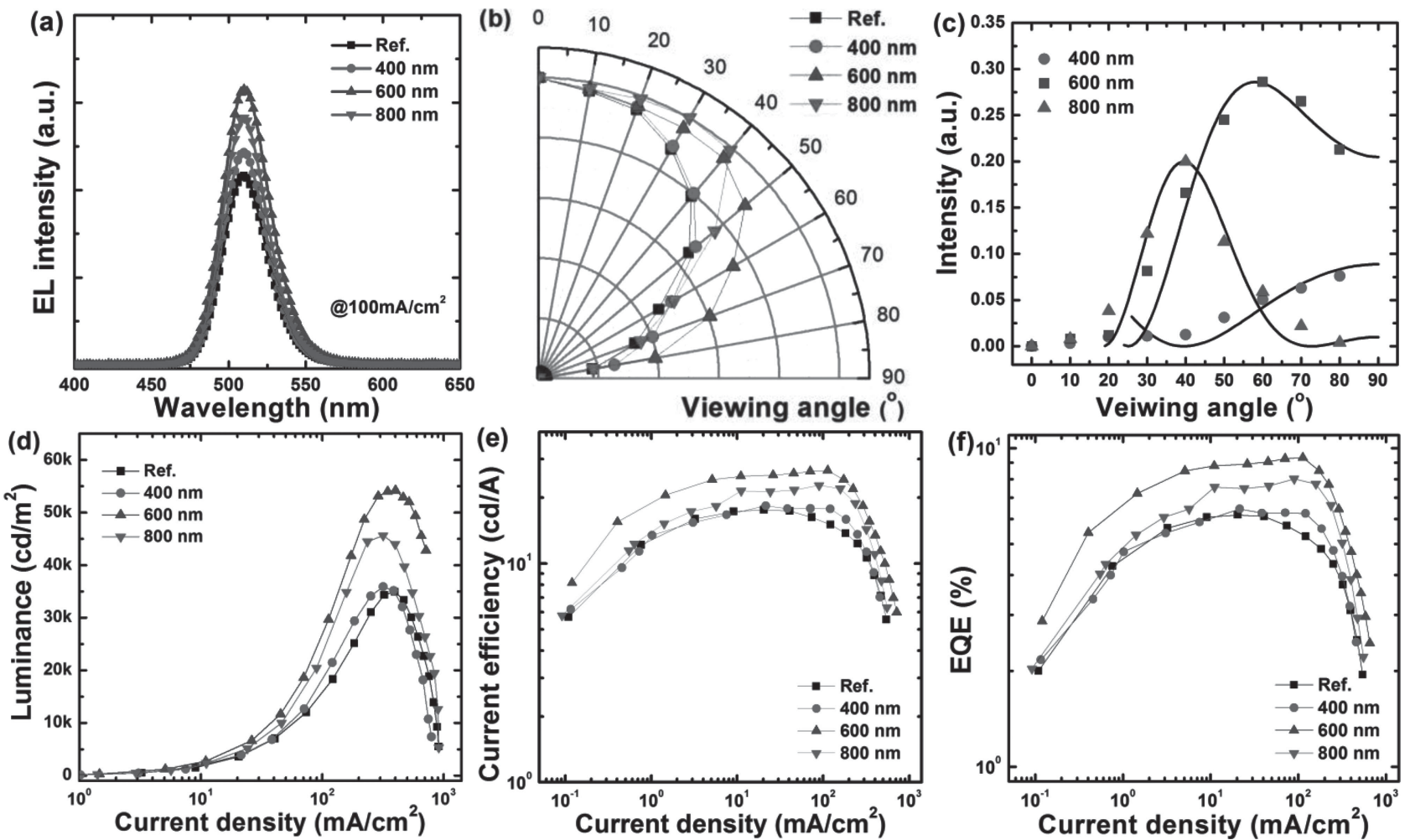

Figure 5. (a) EL spectra of QLEDs acquired from an integrating optical sphere. (b) Angular dependencies of the reference device and the devices with different $\mathrm{ZnO}$ array pitches. (c) Fitting of the angular distribution for QLEDs with different ZnO array pitches. (d) Luminance, (e) current efficiency, and (f) EQE versus current density. 
with the $\mathrm{ZnO}$ pillar array pitches of $600 \mathrm{~nm}$ and $800 \mathrm{~nm}$, however, exhibit quite different angular emission behaviours that present non-Lambertian emission distributions. The emission intensity decreasing for the device with $600 \mathrm{~nm}$ pitch $\mathrm{ZnO}$ pillars is much slower and the device with $800 \mathrm{~nm}$ pitch $\mathrm{ZnO}$ pillars reaches its maximum emission intensity at a viewing angle of $30^{\circ}$. The non-Lambertian emission behaviours for the two QLEDs could be explained by the grating effect of the ordered $\mathrm{ZnO}$ nanostructures. The trapped light in QLEDs can be extracted into air due to the presence of $\mathrm{ZnO}$ nanopillar arrays, and if we assume that incidence angle is $0^{\circ}$, the emission intensity of diffracted light can be written as ${ }^{[26]}$

$\mathrm{f}(\theta)=A \times\left\{\cos \left[\frac{\pi p}{\lambda} \times \sin (\theta)\right]\right\}^{2}$

where $\theta$ presents the emission angle viewed from air, $A$ is the amplitude of light extracted by the grating, $p$ is the pitch of periodic $\mathrm{ZnO}$ nanopillar arrays, and $\lambda$ is the emission wavelength of QLEDs, which is set to the peak wavelength $510 \mathrm{~nm}$ in our case.

The EL intensity of the diffracted light emitted from the QLEDs with different $\mathrm{ZnO}$ array pitches as a function of the emission angle is shown in Figure $5 c$, which is obtained by subtracting the total angular emission for the reference device from that of the corresponding QLEDs. The resulting data are further fitted using Equation (1) and the fitted curves show a good agreement with our experimental results. The constant A for the light extracted by the $\mathrm{ZnO}$ nanopillars, estimated from the fitting curves, is $0.09,0.286$, and 0.2 for QLEDs with the $\mathrm{ZnO}$ pillar array pitch of 400,600 , and $800 \mathrm{~nm}$, respectively. It can be noted that the fitting coefficient for the device with the $400 \mathrm{~nm}$ pitch $\mathrm{ZnO}$ pillars is much smaller, which indicates the weak diffraction grating effect of $\mathrm{ZnO}$ nanopillar arrays. The enhanced device performance for these QLEDs with the $\mathrm{ZnO}$ nanopillar arrays is shown in Figures $5 \mathrm{~d}-\mathrm{f}$. In Figure $5 \mathrm{~d}$, the QLED with the $600 \mathrm{~nm}$ pitch $\mathrm{ZnO}$ pillars exhibits the highest brightness and its luminance reaches $54,200 \mathrm{~cd} / \mathrm{m}^{2}$ at the applied voltage of $11 \mathrm{~V}, 1.5$ folds larger than that of the reference device. Figures 5e and $\mathrm{f}$ present the EQE-J and CE-J curves of device. With the incorporation of $\mathrm{ZnO}$ nanopillars, the device with the $\mathrm{ZnO}$ nanopillar array pitch of $600 \mathrm{~nm}$ made a record
CE of $26.6 \mathrm{~cd} / \mathrm{A}$ at a luminance of $29690 \mathrm{~cd} / \mathrm{m}^{2}$. This corresponds to an EQE level of $9.34 \%$, significantly higher than that of the reference device $(6.17 \%)$, which is enhanced by a factor of $51.4 \%$.

The increase in LEE of QLED structures due to the presence of $\mathrm{ZnO}$ nanopillar arrays is verified using two-dimensional Lumerical finite difference time domain (FDTD) simulation. A $20 \mu \mathrm{m}$ wide simulation area is constructed to include multiple periods of patterning. Physically matched layers are used as the boundary condition to surround the simulation area to absorb any light radiation impinging on it. Dipole light source is placed in the QD emissive layer to replicate the light radiation generated due to electron-hole recombination. The $\mathrm{ZnO}$ nanopillar arrays with a $200 \mathrm{~nm}$ height, a $200 \mathrm{~nm}$ diameter and a $600 \mathrm{~nm}$ pitch are placed atop the $200 \mathrm{~nm}$ thick $\mathrm{ZnO}$ seed layer. The LEE is measured in the wavelength range of 400 to $700 \mathrm{~nm}$ in the far-field after integrating all extracted light radiation in $1^{\circ}$ solid angle, and the LEE enhancement in QLEDs is obtained by dividing the total power coming out of the device with $\mathrm{ZnO}$ nanopillar arrays (in air) to the total power for the reference device. As shown in Figure 6a, most of the light that is generated in the QD emissive region suffers the total internal reflection from the glass-air interface and is trapped within the QLED architecture. In contrast, Figure 6b shows the light escaping out from the QLED due to the presence of $\mathrm{ZnO}$ nanopillar arrays on top of the glass surface, which thus significantly enhances the LEE of QLEDs. It can be clearly quantified from the data in Figure $6 c$ that the LEE enhancement is $\sim 1.5$ fold at $510 \mathrm{~nm}$ emission wavelength compared to the reference device, which is in excellent agreement with our experimental findings.

A QLED architecture constituting multiple organic and inorganic thin layers with varying indices behaves as a LED microcavity, ${ }^{[28-30]}$ in which the light radiation is partially reflected and transmitted through different multilayers depending on their refractive indices when the photon is emitted from the QD layer. Therefore, the performance of the resulting QLEDs can be also affected by the microcavity structure of device. In a QLED microcavity, thick metal electrode at one end of the QLED device serves as one of the reflecting mirrors, whereas different organic and inorganic multilayers act as partial reflectors at the other end. It is worth mentioning that QLED architecture has more distinctive refractive indices compared with
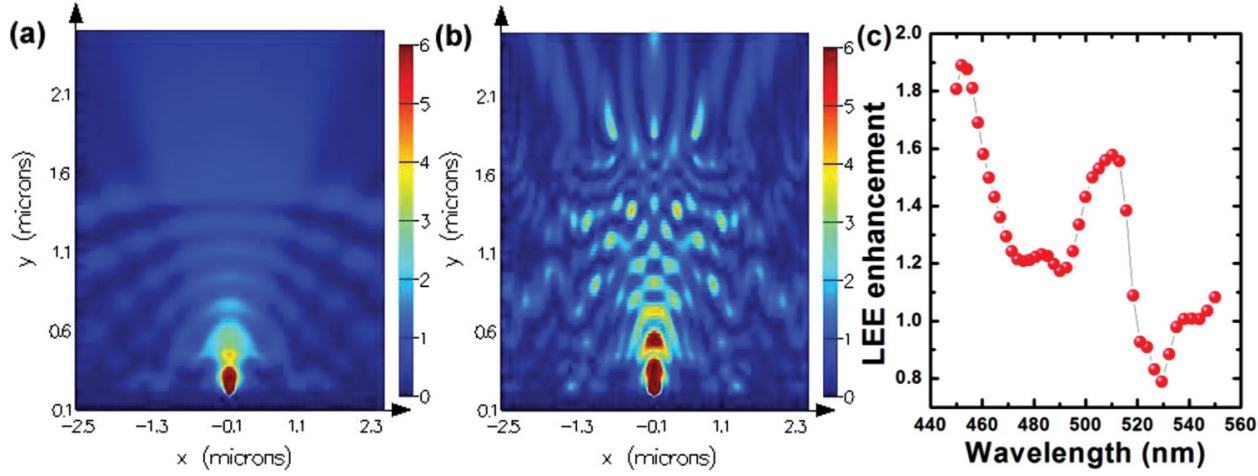

Figure 6. FDTD results showing (a) light trapped inside the glass substrate due to the reflection from the glass-air interface and (b) light escaping into air in the presence of periodic $\mathrm{ZnO}$ nanopillar arrays on top of the glass surface of QLEDs. (c) LEE enhancement in the QLED with the ZnO nanopillar array pitch of $600 \mathrm{~nm}$. 
OLEDs due to the inorganic QD and ZnO layers, which have higher refractive indices over organic materials. To explore the microcavity effects in the resulting QLEDs, the 2D-QLED structure without the incorporation of $\mathrm{ZnO}$ nanopillar arrays is designed in Lumerical FDTD (Figure 7). The device microcavity structure allows the formation of a standing wave within the cavity. This means that the electric field intensity profile along the length of the microcavity is not constant and has nodes and peaks (antinodes). The position of QD layer must align with the peak of electric field intensity profile within the microcavity structure for obtaining high-performance QLEDs, which indicates that the optical density of states should be high over the QD emissive layer. If the position of QD layer lines up with the node of electric field intensity profile, the spontaneous emission from the QD layer is suppressed rather than enhanced. In the designed simulation, the QLED microcavity is illuminated with a plane wave source with wavelength varying from 400 to $700 \mathrm{~nm}$. The total electric field inside the microcavity is visualised using a 2D frequency domain field profile monitor. This monitor helps in imaging the position of cavity modes within the optical microcavity. Similarly, the refractive index of each layer is monitored using refractive index monitor. Periodic boundary conditions are used in the $\gamma$-direction for $10 \mu \mathrm{m}$ wide FDTD simulation area, while a physically matched layer is used at the top and a metal boundary condition is used at the bottom. Figure 7a shows the 2D-FDTD refractive indices of the multi-layered QLED structure and the variation of total electric field intensity (standing wave) within the QLED microcavity. The FDTD simulation results given in Figure $7 \mathrm{~b}$ clearly show that the position of the QD emissive layer in our QLED (from 0.24 to $0.27 \mu \mathrm{m}$ along the $\gamma$-axis) quite matches with the peak of total electric field and hence enhances the emission of photons. In addition, it is found that the cavity mode can also increase the LEE of green-emitting QLEDs by highly optically transmitting in a particular wavelength range (green range) while suppressing other wavelengths, which is confirmed by measuring the reflection from the QLED microcavity using the same FDTD simulation. The 1D frequency-domain monitor is positioned behind the plane wave source to measure this reflection. As shown in Figure 7c, the QLED microcavity suppresses the reflection around $500 \mathrm{~nm}$ optical wavelength, and thus gives high transmission for the green wavelength.
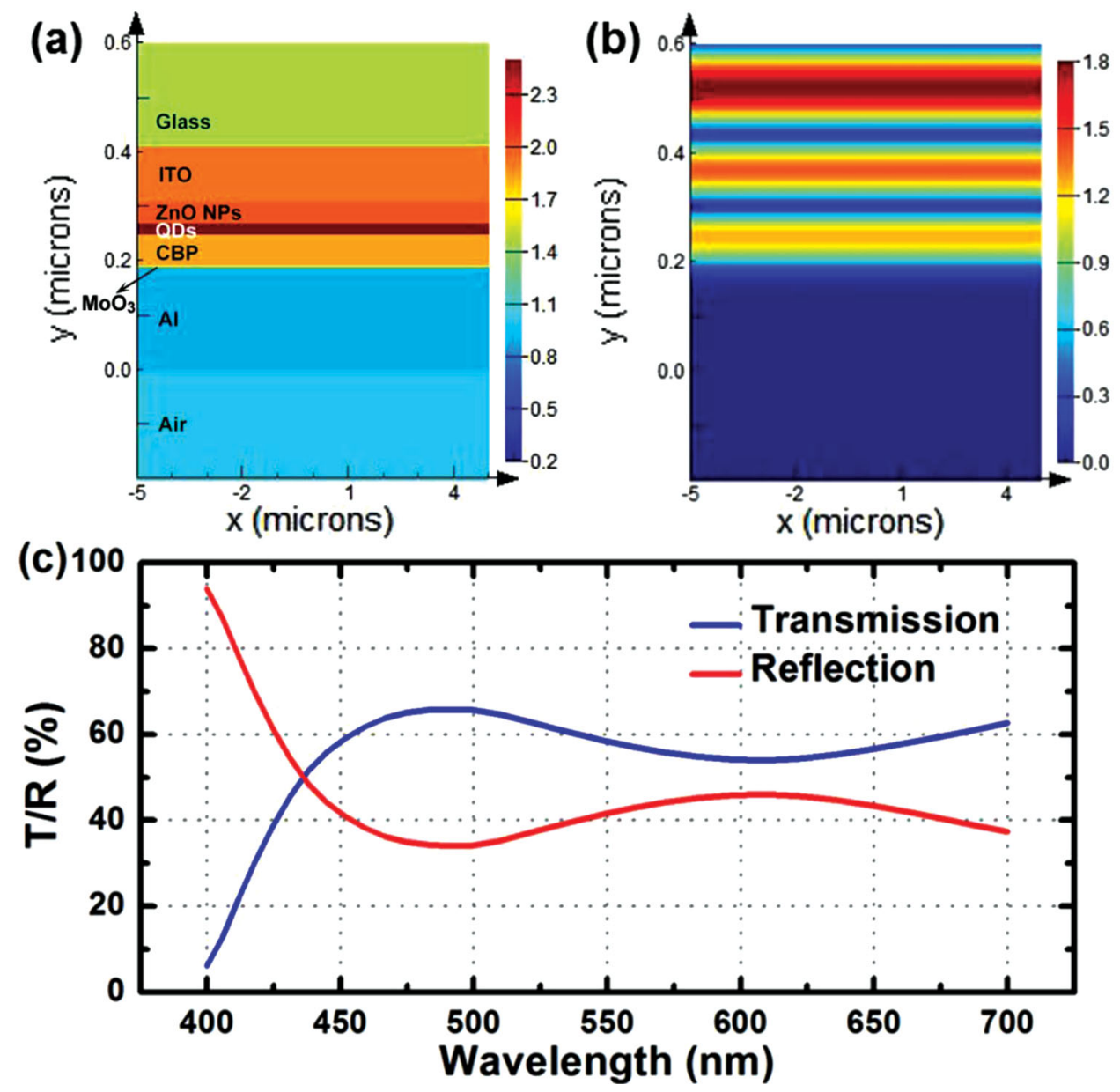

Figure 7. (a) Refractive indices of different organic and inorganic layers of QLEDs (from the top to the bottom: Glass/ITO/ZnO NPs/QDs/CBP/MoO $/$ Al). (b) Total electric field intensity at $510 \mathrm{~nm}$ wavelength within the QLED microcavity simulated using Lumerical FDTD Solutions. (c) Optical reflection and transmission spectra for the QLEDs. 


\section{Conclusions}

We demonstrated for the first time the dramatically enhanced light extraction in QLEDs based on a layer of large-scale, periodic $\mathrm{ZnO}$ nanopillar arrays faricated using a simple and efficient PRINT method. The resulting QLED with a $\mathrm{ZnO}$ nanopillar array pitch of $600 \mathrm{~nm}$ showed a record CE of $26.6 \mathrm{~cd} / \mathrm{A}$. The maximum EQE of $9.35 \%$ achieved in the present work is also the highest EQE value ever reported for green QLEDs to date, which corresponds to $51.3 \%$ efficiency enhancement in the total light output compared with the reference device. Lumerical FDTD simulations reveal that the significant light outcoupling efficiency improvement results from the effective diffraction of the generated light, which is otherwise trapped in the glass mode, in the presence of $\mathrm{ZnO}$ nanopillar arrays and microcavity effects in the inverted QLEDs. These findings suggest that the cost-effective $\mathrm{ZnO}$ nanopillars are promising light outcoupling layer for improving QLED performance and this approach presented here may have important implications for understanding and designing high-performance QLEDs by enhancing light extraction efficiency.

\section{Experimental Section}

Preparation of $\mathrm{ZnO}$ Nanopillar Arrays: The large-scale, periodic $\mathrm{ZnO}$ nanopillar arrays were prepared on the glass surface of patterned ITOglass substrate according to a modified pattern replication approach of non-wetting templates technique, ${ }^{[26]}$ in which a $\mathrm{ZnO}$ thin film of $20 \mathrm{~nm}$ is first deposited on the glass surface as a $\mathrm{ZnO}$ seed layer by RF magnetron sputtering for obtaining high-quality $\mathrm{ZnO}$ nanopillar arrays. Next, a polydimethylsiloxane (PDMS) mold was prepared using the patterned silicon master substrate. The PDMS mold was then pressed on the glass surface of substrate that was coated with $\mathrm{ZnO}$ precursor

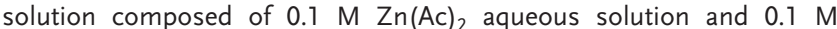
diethanolamine (DEA) by drop-casting method. The ITO-glass substrate was heated for removing solvent. Subsequently, the mold was peeled off, followed by annealing at $180^{\circ} \mathrm{C}$ for $15 \mathrm{~min}$, and the as-prepared ITOglass substrate with ordered $\mathrm{ZnO}$ nanopillar arrays could be obtained.

Synthesis of CdSe/ZnS QDs and ZnO Nanoparticles: CdSe/ZnS QDs with chemical-composition gradients were prepared according to a method reported in the literature. ${ }^{[31]}$ For a typical preparation of green-emitting QDs, $0.1 \mathrm{mmol}$ of cadmium oxide (CdO), $4 \mathrm{mmol}$ of zinc acetate $\left(\mathrm{Zn}(\text { Acet })_{2}\right)$, and $5 \mathrm{~mL}$ of oleic acid $(\mathrm{OA})$ were loaded in a $50 \mathrm{~mL}$ 3-neck flask, heated to $150^{\circ} \mathrm{C}$ under vacuum to form cadmium oleate $\left(\mathrm{Cd}(\mathrm{OA})_{2}\right)$ and zinc oleate $\left(\mathrm{Zn}(\mathrm{OA})_{2}\right)$. Subsequently, $20 \mathrm{~mL}$ of 1-octadecene (1-ODE) was added to the reaction flask, and the reactor was then filled with nitrogen and heated up to $300{ }^{\circ} \mathrm{C}$. At the elevated temperature, $1.6 \mathrm{~mL}$ of tri-n-octylphosphine (TOP) with $0.15 \mathrm{mmol}$ of selenium (Se) and $4 \mathrm{mmol}$ of sulphur (S) dissolved was injected into the flask swiftly. The reaction mixture was maintained at $300^{\circ} \mathrm{C}$ for $10 \mathrm{~min}$ for the QD growth. To purify the synthesized QDs, the reaction mixture was cooled down to room temperature, and the QDs were extracted by the addition of acetone and methanol, followed by centrifugation. The as-prepared QDs can be readily dispersed in toluene. The purified QDs with an average particle size of $\sim 7 \mathrm{~nm}$ was measured to give a quantum yield of $\sim 60 \%$ in toluene by comparing the fluorescence intensities with a standard reference dye, fluorescein-27. ZnO nanoparticles (NPs) were synthesized with a mean diameter of 3-4 nm based on a solution-precipitation process, which was previously reported in detail.[21]

Fabrication of QLED Devices: The patterned ITO substrates were cleaned by sonication sequentially in detergent, de-ionized water, acetone, and isopropyl alcohol. The ZnO NP layer was then spin-coated on the ITO substrate with $\mathrm{ZnO}$ nanopillar arrays from $\mathrm{ZnO}$ ethanol solution at $1000 \mathrm{rpm}$ for $60 \mathrm{~s}$ and annealed at $150{ }^{\circ} \mathrm{C}$ for $30 \mathrm{~min}$ in a nitrogen glove box. Next, the QD layer was deposited on ZnO NP layer by spin-coating the QD dispersion ( $15 \mathrm{mg} / \mathrm{mL}$ in toluene) at a rate of $2000 \mathrm{rpm}$ for $60 \mathrm{~s}$, and cured at $90{ }^{\circ} \mathrm{C}$ for $30 \mathrm{~min}$. After that the 4,4'-N, $N^{\prime}$-dicarbazole-biphenyl (CBP, $\left.60 \mathrm{~nm}\right), \mathrm{MoO}_{3}(10 \mathrm{~nm})$, and Al $(190 \mathrm{~nm})$ layers were thermally deposited under a base pressure of $\sim 2 \times 10^{-4} \mathrm{~Pa}$

Instrumentation: The EL spectra of the QLEDs were measured using a Photo Research PR705 Spectra Scan spectrometer, while the luminance-current-voltage characteristics of the devices were measured simultaneously with a programmable Yokogawa GS610 source meter and a Konica Minolta luminance meter LS-110 in air at room temperature. The integral EL spectra was measured using an integrating optical sphere and the measurement of overall device performances for QLEDs with ZnO nanopillars were made using a technique described by Forrest et al. ${ }^{[32]}$ in which a photodiode was placed in contact with the active pixel (for which the area of the photodiode is much larger than that of the active pixel). The EL emission from QLEDs was first measured as the photodiode current and the current of device was also obtained simultaneously. The total device lumiance and efficiency were calculated by these two quantities together with the integral $\mathrm{EL}$ spectra. The PL spectra were recorded using a Shimadzu RF-5301PC spectrofluorophotometer equipped with a $150 \mathrm{~W}$ Xenon lamp as the excitation source. The morphologies of ordered $\mathrm{ZnO}$ nanopillars were observed using scanning electron microscopy (SEM, JEOL, JSM-5910) and field emission scanning electronic microscopy (FE-SEM, JEOL, JSM6340F). All measurements were carried out at room temperature under ambient conditions.

\section{Acknowledgements}

The authors would like to thank the financial supports from Singapore National Research Foundation under NRF-RF-2009-09 and NRF-CRP-6-2010-02 and the Science and Engineering Research Council, Agency for Science, Technology and Research (A*STAR) of Singapore (project Nos. 0921010057 and 112120 2009).

Received: January 19, 2014

Revised: April 20, 2014

Published online: July 22, 2014

[1] O. Chen, J. Zhao, V. P. Chauhan, J. Cui, C. Wong, D. K. Harris, H. Wei, H.-S. Han, D. Fukumura, R. K. Jain, M. G. Bawendi, Nat. Mater. 2013, 12, 445-451.

[2] H. Shen, X. Bai, A. Wang, H. Wang, L. Qian, Y. Yang, A. Titov, J. Hyvonen, Y. Zheng, L. S. Li, Adv. Funct. Mater. 2014, 24, 2367.

[3] C. B. Murray, D. J. Norris, M. G. Bawendi, J. Am. Chem. Soc. 1993 , $115,8706-8715$.

[4] J. S. Steckel, J. P. Zimmer, S. Coe-Sullivan, N. E. Stott, V. Bulović, M. G. Bawendi, Angew. Chem. Int. Ed. 2004, 43, 2154-2158.

[5] W. K. Bae, K. Char, H. Hur, S. Lee, Chem. Mater. 2008, 20, 531-539.

[6] X. Yang, D. Zhao, K. S. Leck, S. T. Tan, Y. X. Tang, J. Zhao, H. V. Demir, X. W. Sun, Adv. Mater. 2012, 24, 4180-4185.

[7] J. Lim, S. Jun, E. Jang, H. Baik, H. Kim, J. Cho, Adv. Mater. 2007, 19, 1927-1932.

[8] B. S. Mashford, M. Stevenson, Z. Popovic, C. Hamilton, Z. Zhou, C. Breen, J. Steckel, V. Bulović, M. Bawendi, S. Coe-Sullivan, P. T. Kazlas, Nature Photon. 2013, 7, 407-412.

[9] Y. Shirasaki, G. J. Supran, M. G. Bawendi, V. Bulović, Nature Photon. 2013, 7, 13-23.

[10] T.-H. Kim, K.-S. Cho, E. K. Lee, S. J. Lee, J. Chae, J. W. Kim, D. H. Kim, J.-Y. Kwon, G. Amaratunga, S. Y. Lee, B. L. Choi, Y. Kuk, J. M. Kim, K. Kim, Nature Photon. 2011, 5, 176-182. 
[11] T.-H. Kim, D.-Y. Chung, J. Ku, I. Song, S. Sul, D.-H. Kim, K.-S. Cho, B. L. Choi, M. J. Kim, S. Hwang, K. Kim, Nat. Commun. 2013, DOI: $10.1038 /$ ncomms3637.

[12] J. Lim, M. Park, W. K. Bae, D. Lee, S. Lee, C. Lee, K. Char, ACS Nano 2013, 7, 9019-9026.

[13] G. J. Supran, Y. Shirasaki, K. W. Song, J.-M. Caruge, P. T. Kazlas, S. Coe-Sullivan, T. L. Andrew, M. G. Bawendi, V. Bulović, MRS Bull. 2013, 38, 703-711.

[14] X. Yang, E. Mutlugun, Y. Zhao, Y. Gao, K. S. Leck, Y. Ma, L. Ke, S. T. Tan, H. V. Demir, X. W. Sun, Small 2013, DOI: 10.1002/ smll.201301199.

[15] Z. Tan, Y. Zhang, C. Xie, H. Su, J. Liu, C. Zhang, N. Dellas, S. E. Mohney, Y. Wang, J. Wang, J. Xu, Adv. Mater. 2011, 23, 3553-3558.

[16] Z. Chen, B. Nadal, B. Mahler, H. Aubin, B. Dubertret, Adv. Funct. Mater. 2014, 24, 295.

[17] R. Meerheim, M. Furno, S. Hofmann, B. Lüssem, K. Leo, Appl. Phys. Lett. 2010, 97, 253305.

[18] S.-Y. Kim, J.-J. Kim, Org. Electron. 2010, 11, 1010-1015.

[19] J. Kwak, W. K. Bae, D. Lee, I. Park, J. Lim, M. Park, H. Cho, H. Woo, D. Y. Yoon, K. Char, S. Lee, C. Lee, Nano Lett. 2012, 12, 2362-2366.

[20] E. L. Ratcliff, P. A. Veneman, A. Simmonds, B. Zacher, D. Huebner, S. S. Saavedra, N. R. Armstrong, Anal. Chem. 2010, 82, $2734-2742$.
[21] L. Qian, Y. Zheng, J. Xue, P. H. Holloway, Nature Photon. 2011, 5, 543-548.

[22] W. H. Koo, S. M. Jeong, F. Araoka, K. Ishikawa, S. Nishimura, T. Toyooka, H. Takezoe, Nature Photon. 2010, 4, 222-226.

[23] C. Lee, J.-J. Kim, Small 2013, 9, 3858-3863.

[24] J. H. Hwang, T. H. Park, H. J. Lee, K. B. Choi, Y. W. Park, B.-K. Ju, Opt. Lett. 2013, 38, 4182-4185.

[25] H. Jeong, D. J. Park, H. S. Lee, Y. H. Ko, J. S. Yu, S.-B. Choi, D.-S. Lee, E.-K. Suh, M. S. Jeong, Nanoscale 2014, 6, 4371-4378.

[26] S. W. Liu, J. X. Wang, Y. Divayana, K. Dev, S. T. Tan, H. V. Demir, X. W. Sun, Appl. Phys. Lett. 2013, 102, 053305.

[27] Z. Kyaw, W. Jianxiong, K. Dev, S. T. Tan, Z. Ju, Z.-H. Zhang, Y. Ji, N. Hasanov, W. Liu, X. W. Sun, H. V. Demir, Opt. Express 2013, 21, 26846-26853.

[28] H. K. Kim, S.-H. Cho, J. R. Oh, Y.-H. Lee, J.-H. Lee, J.-G. Lee, S.-K. Kim, Y.-I. Park, J.-W. Park, Y. R. Do, Org. Electron. 2010, 11, 137-145.

[29] D. Poitras, C.-C. Kuo, C. Py, Opt. Express 2008, 16, 8003-8015.

[30] W. C. H. Choy, C. Y. Ho, Opt. Express 2007, 15, 13288-13294.

[31] W. K. Bae, J. Kwak, J. Lim, D. Lee, M. K. Nam, K. Char, C. Lee, S. Lee, Nano Lett. 2010, 10, 2368-2373.

[32] S. R. Forrest, D. D. C. Bradley, M. E. Thompson, Adv. Mater. 2003, 15, 1043-1048. 